\title{
Stage IIIB Prostate Cancer AJCC v8
}

National Cancer Institute

\section{Source}

National Cancer Institute. Stage IIIB Prostate Cancer A/CC v8. NCI Thesaurus. Code C140171.

Stage IIIB includes: T3-4, N0, M0, PSA: Any, Grade Group 1-4; T3: Prostate cancer with extraprostatic tumor that is not fixed or does not invade adjacent structures. T4:

Prostate cancer in which the tumor is fixed or invades adjacent structures other than seminal vesicles such as external sphincter, rectum, bladder, levator muscles, and/or pelvic wall. N0: Prostate cancer with no positive regional nodes. M0: Prostate cancer without evidence of distant metastasis. Grade Group 1: Gleason Score 6 or less, Gleason Pattern 3 or less+3. Grade Group 2: Gleason Score 7, Gleason Pattern 3+4. Grade Group 3: Gleason Score 7, Gleason Pattern 4+3. Grade Group 4: Gleason Score 8, Gleason Pattern 4+4. (AJCC 8th ed.) 